\title{
Neurosarcoidosis: An Illustrative Case and Review of Perioperative Considerations
}

\author{
Vivek Arora, Srilatha Mallu
}

Department of Anesthesiology, Henry Ford Hospital, Detroit, USA.

Email: vivek_cdg@hotmail.com

Received June 21 ${ }^{\text {st }}$, 2012; revised July 22 ${ }^{\text {nd }}, 2012$; accepted August $16^{\text {th }}, 2012$

\begin{abstract}
Sarcoidosis is a multisystem inflammatory granulomatous disease of unknown etiology. Neurosarcoidosis (NS) is a rare but potentially devastating manifestation of sarcoidosis, with a prevalence of approximately $5 \%$ in patients with systemic sarcoidosis. Due to the possible involvement of any part of the nervous system, a myriad of neurological manifestations can occur. Clinical features resulting from involvement of the hypothalmo-pituitary axis and cranial nerves, in particular, cranial nerve VII are the more common presentations of this condition. Medical therapy with corticosteroids is the mainstay of treatment and providing tissue for diagnosis remains the principal indication for surgery. Therapeutically, neurosurgery is indicated only for life-threatening complications. We describe the clinical case of a patient with fatally progressive NS who had multiple anesthetic exposures. This case highlights the perioperative considerations of NS and its anesthetic implications.
\end{abstract}

Keywords: Neurosarcoidosis; Sarcoidosis; Granuloma; Perioperative Considerations

\section{Introduction}

Sarcoidosis is a multisystem inflammatory granulomatous disease of unknown etiology. The incidence of sarcoidosis in North America is estimated at 3 - 10 per 100,000 among Caucasians and 35 - 80 per 100,000 among African Americans [1]. Neurosarcoidosis is a rare but potentially devastating manifestation of sarcoidosis, with a prevalence of approximately 5\% in patients with systemic sarcoidosis. However the incidence of subclinical or undiagnosed neurological involvement in patients with sarcoidosis is much higher, upto 26\% [2]. Clinical features resulting from involvement of the hypothalmopituitary axis and cranial nerves, in particular cranial nerve VII, are the more common presentations of this condition [3]. Medical therapy with corticosteroids is the mainstay of treatment and providing tissue for diagnosis remains the principal indication for surgery [4]. Therapeutically, neurosurgery is indicated only for life threatening complications such as increased intracranial pressure secondary to hydrocephalus. Surgical resection of mass lesions in the brain or spinal cord is rarely undertaken [5]. Occasionally patients, with underlying NS, may present for surgical procedures unrelated to underlying sarcoidosis.

We describe the clinical case of a patient with fatally progressive NS who had multiple anesthetic exposures, initially for a diagnostic MRI, then for a diagnostic brain biopsy and later for insertion followed by several revisions of a ventriculoperitoneal shunt. This case highlights the perioperative considerations of NS and its anesthetic implications.

\section{Case Report}

A 34-year-old male patient (height $182 \mathrm{~cm}$, weight 160 $\mathrm{kg}$ ) with a history of hypertension and type II diabetes mellitus was admitted to our hospital in 2005 with acute onset, progressive lower extremity weakness. Computed tomography of the chest revealed hilar lyphadenopathy and abnormal leptomeningeal enhancement in the region of the infundibulum and contrast enhanced magnetic resonance imaging (MRI) of the brain demonstrated intraventricular enhancement of the third ventricular on (Figure 1). Contrast-enhanced sagittal T1-weighted imaging of the cervical spine showed linear and multiple nodular enhancing lesions along the surface of the spinal cord and spinal nerve roots (Figure 2). Based on the MRI findings a temporal craniotomy was performed and meningeal and brain biopsies were obtained. The perioperative course was uneventful. Histopathological examination revealed perivascular, non-caseating granulomatous inflammation and a definitive diagnosis of NS was made. Disease symptoms partially responded to high dose intravenous corticosteroids and the patient was able to ambulate with support. He was discharged on oral 
prednisone for disease suppression and phenytoin for seizure prophylaxis. Hormonal evaluation except for known diabetes was normal at discharge. Over the next two years, audiovestibular and hypothalamo-pituitary axis involvement was noted and patient developed unilateral hearing loss, and hypothyroidism, hypogonadism, hypoadrenalism and diabetes insipidus requiring levothyroxine, testosterone continuation of corticosteroids and desmopressin respectively. Disease progression continued, and methotrexate and mycophenolate mofetil were added to the treatment regimen. Later on the patient developed acute symptomatic seizures, which were treated initially with phenytoin and subsequently with levitrictam. Peripheral nervous system involvement was noted in the form of sensory neuropathy requiring treatment with gabapentin. In 2007 the patient presented with diffuse encephalopathy and non-communicating hydrocephalus necessitating a ventricular peritoneal (VP) shunt insertion. Preoperative renal and hepatic function tests were reviewed and were within normal limits. Hormone replacement therapy was continued and supplementary steroids were administered preoperatively. Fluid status and serum electrolytes were closely monitored and appropriately replaced. Intraoperative course was uneventful but in the post operative period patient developed hyponatremia. Desmopressin was temporarily withheld and hyponatremia was managed with fluid restriction. Subsequently patient underwent multiple revisions of his VP shunt. In late 2009 patient died from severe sepsis secondary to skin and a urinary tract infection.

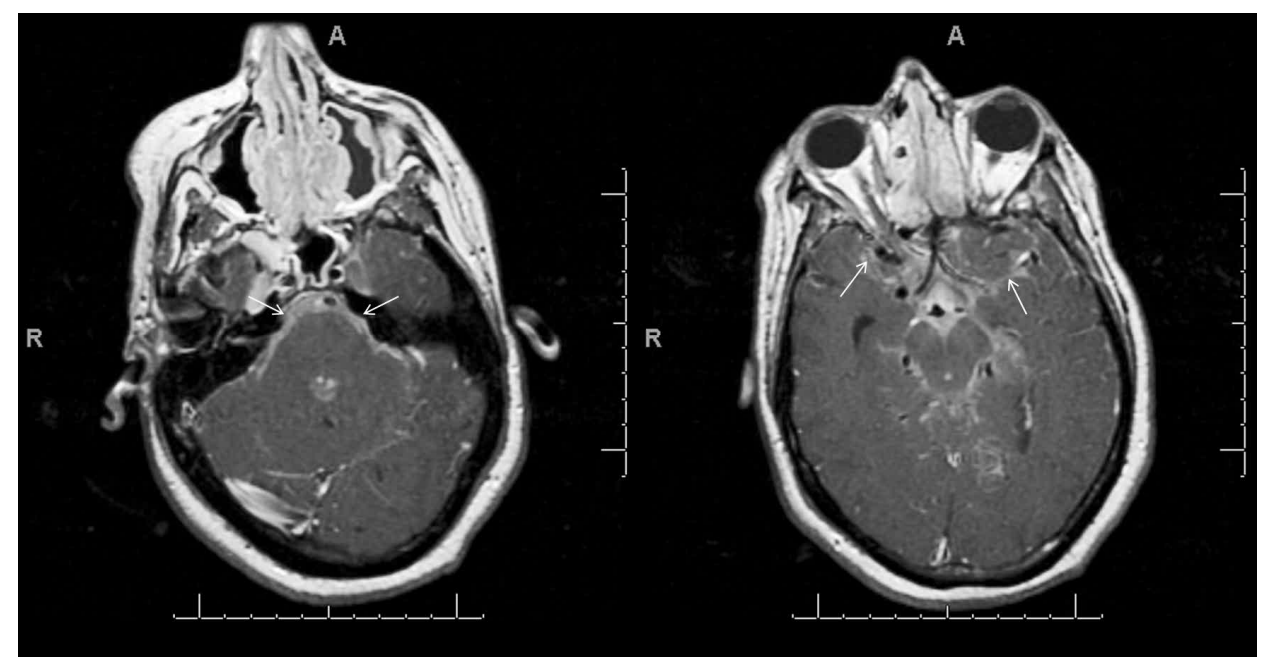

Figure 1. Leptomeningeal sarcoidosis of brain. T1-weighted axial images from caudal to cranial (A, B), obtained after contrast administration shows enhancement involving basilar cisterns, Sylvian fissures and cortical sulci (white arrows).

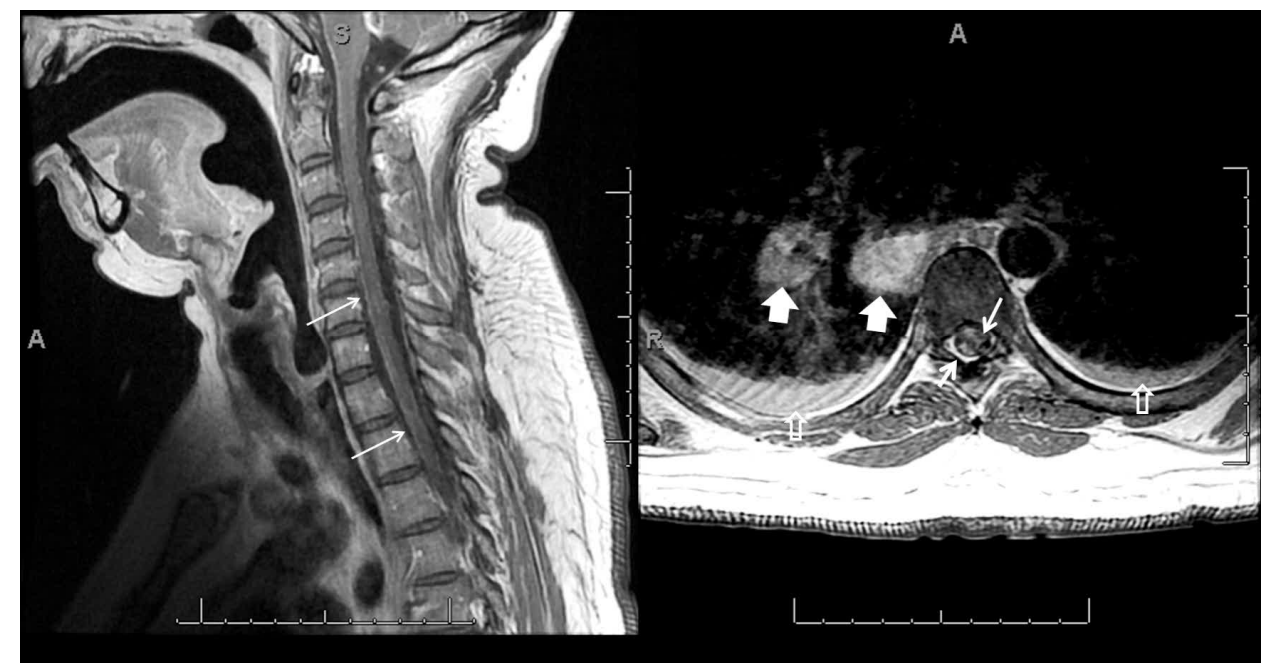

Figure 2. Spinal leptomeningeal sarcoidosis. Contrast-enhanced sagittal T1-weighted image of the cervical spine shows linear and multiple nodular enhancing lesions along surface of spinal cord and on spinal nerve roots (thin white arrows). Post contrast axial T1-weighted image of the thoracic spine demonstrates nodular leptomeningeal deposits (thick white arrows) with enlarged subcarinal and left hilar lymph nodes (arrowheads) and bilateral pleural effusion (hollw white arrows). 


\section{Discussion}

\subsection{Epidemiology/Pathogenesis}

Sarcoidosis is a ubiquitous disease seen worldwide. The overall disease prevalence in the United States is estimated at 40 per 100,000 with an annual incidence of $0.035 \%$ to $0.080 \%$ among African Americans and 0.003\% to $0.01 \%$ in whites [1]. As for sarcoidosis confined solely to the nervous system, the annual incidence is estimated at less than 0.2 per 100,000 among whites [6]. Spinal cord involvement occurs in less than 5\% of these patients. Asymptomatic central nervous system (CNS) involvement is found in a much higher proportion of patients and emulates the finding in other organs such as the heart, liver, spleen, and lung where sarcoidal involvement is often occult and asymptomatic [7]. Neurological symptoms are the presenting manifestation in half of the patients with NS and in the remaining half, symptoms appear within 2 years of being diagnosed with systemic sarcoidosis [7].

The pathologic hallmark of sarcoidosis is the noncaseating granuloma. Activated $\mathrm{T}$ cells and macrophages release interferon gamma, interleukin 2 and other proinflammatory factors that mediate the formation of theses granulomas. As the disease progresses, persistence of the inflammatory process induces fibrotic changes resulting in irreversible tissue damage.

\subsection{Clinical Course and Diagnosis}

Neurological manifestations (Table 1) occur as a part of the spectrum of the systemic disease that consists of, but is not limited to, involvement of the lungs, skin, eyes, heart, and kidney. Cranial neuropathies are identified in $50 \%$ to $75 \%$ of patients with facial and optic nerve involvement being the most common [7]. Audiovestiblar dysfunction is rare and when present is usually bilateral and causes sensorineural hearing loss [8]. In addition to these solitary or multiple involvements of almost all cranial nerves has been reported in patients with NS [3]. Leptomeningeal involvement is seen in $10 \%$ to $20 \%$ of patients and may present as aseptic meningitis or hydrocephalus. Approximately 50\% of patients with a diagnosis of NS experience parenchymal brain disease. Based on the anatomical location of the sarcoidal granulomas patients may present with a multitude of symptoms or syndromic features (Table 1). NS has a predilection for the base of the brain and $10 \%-15 \%$ of patients develop neuroendocrine related symptoms from hypothalamic and pituitary gland involvement resulting in diabetes inspidus, adenopituitary failure or amenorrhea-galactorrhea syndrome. Diffuse parenchymal involvement may

Table 1. Neurological manifestations of sarcoidosis [3,7].

\begin{tabular}{|c|c|}
\hline Neurological Features & \\
\hline Cranial Neuropathy & $50 \%-75 \%$ \\
\hline 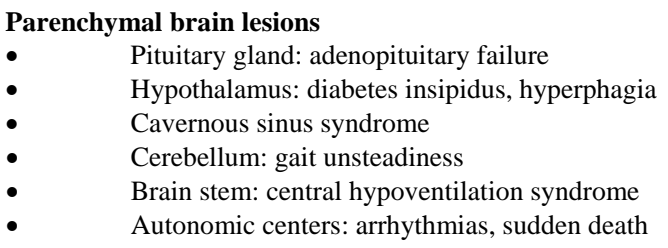 & $50 \%$ \\
\hline Cognitive/behavioral manifestations & $20 \%$ \\
\hline Meningeal disease & $10 \%-20 \%$ \\
\hline Seizures & $5 \%-10 \%$ \\
\hline $\begin{array}{l}\text { Spinal lesions } \\
\text { - } \quad \text { paresthesias, paraparesis, tetraparesis } \\
\text { - } \\
\text { - } \\
\text { - } \\
\text { adicular symptoms } \\
\text { cauda equine syndrome }\end{array}$ & $5 \%-10 \%$ \\
\hline $\begin{array}{l}\text { Peripheral neuropathy } \\
\text { - } \quad \text { mononeuropathy, polyradculopahthy } \\
\text { - } \quad \text { Guilain-Barré-like syndrome } \\
\text { - } \quad \text { symmetrical distal polyneuropathy }\end{array}$ & $15 \%$ \\
\hline Myopathy (symptomatic) & $1.4 \%-2.3 \%$ \\
\hline
\end{tabular}


result in encephalopathy ( $5 \%$ - 10\%), psychiatric symptoms $(19 \%)$ and/or seizures (5\% - 10\%). Granulomatous cerebral angitis has also been reported in NS [3]. Unexpected death due to CNS involvement resulting in arrhythmias from infiltration of autonomic centers, epilepsy and obstructive hydrocephalus from brainstem involvement has also been reported [9].

Spinal cord involvement is seen in $10 \%$ of patients. Most clinically significant sarcoidal granulomas are intramedullary [10]. They have an affinity for the cervical level and tend to extend over multiple cord segments [11]. Patients with spinal sarcoidosis may present with insidious, progressive paresthesias and weakness that can progress to paraplegia. Rare cases of cauda equina syndrome secondary to intradural but extramedullary sarcoid involvement have been reported and may be the initial presentation of NS [10].

Peripheral nervous system dysfunction is seen in 15\% of patients with NS [12]. Sarcoidosis can affect any area of the peripheral nervous system and result in mononeuropathy, mononeuritis multiplex or polyneuropathy. Gullan-Barre syndrome, restless leg syndrome in addition to isolated sensory neuropathy and autonomic dysfunction have been reported in patients with systemic sarcoidosis [3].

\subsection{Pharmacotherapy}

As there is a dearth of prospective, randomized, controlled trials in NS current treatment strategies are not standardized and are based on the treatment of systemic sarcoidosis. Corticosteroids remain the mainstay of treatment [4]. In the event that treatment with corticosteroids fails or needs to be discontinued, non steroidal immunosuppressant's (methotrexate, mycophenolate mofetil, cyclophosphamide, azathioprine, chloroquine and hydroxychloroquine, thalidomide, and infliximab) can be added to the treatment regimen (Table 2) [13]. Except for infliximab, the response to alternative immunosuppressants is relatively slow and therefore, in acute life threatening situations high dose intravenous corticosteroids remain the preferred treatment [13].

\subsection{Operative Considerations}

Obtaining tissue for histopathological diagnosis remains the primary indication for surgery. Although, positive central nervous system (dura, brain) histology helps establish a definitive diagnosis, a probable diagnosis of NS can be made based on positive histology from non central nervous system involved organs in the presence of laboratory or radiological evidence compatible with NS [14]. Mediastinoscopy is commonly performed to obtain tissue from lung/hilar lymph nodes. Therapeutically neurosurgery is indicated only when medical management fails and in life threatening emergency situations such as elevated intracranial pressure secondary to hydrocephalus requiring ventricular drainage [15]. Although surgical resection of intracranial and intramedullary spinal mass lesions has been reported overall results remain unsatisfactory [11]. Other surgical procedures reported to have been performed in patients with NS are listed below (Table 3).

Table 2. Medical therapy for neurosarcoidosis [7,14].

\begin{tabular}{|c|c|}
\hline Therapy & Adverse Reactions \\
\hline Prednisone & $\begin{array}{l}\text { Hypertension, adrenal suppression, hyperglycemia, psychosis, insomnia, gastritis, weight } \\
\text { gain, osteoporosis }\end{array}$ \\
\hline Methylprednisone & As above \\
\hline Methotrexate & Hepatotoxicity, interstitial pneumonitis, bone marrow suppression, anemia \\
\hline Mycophenolte mofetil & Leukopenia, anemia, esophagitis, gastritis, gastrointestinal hemorhage \\
\hline Azothioprine & Bone marrow suppression, hypersensitivity syndrome, risk for lymphoma or leukemia \\
\hline Chloroquine, hydroxychloroquine & Irreversible retinal damage, seizures, deafness, tinnitus, alopecia, hypertension, ECG changes \\
\hline Infliximab & $\begin{array}{l}\text { Anaphylaxis, risk for infection, rash, hepatotoxicity, pancytopenia, delayed allergic reactions, } \\
\text { CHF exacerbation, CNS demyelination/inflammation }\end{array}$ \\
\hline Cyclophosphamide & Hemorrhagic cystitis, amenorrhea, azotemia, cardiac toxicity, infertility, neutropenia \\
\hline Radiotherapy & $\begin{array}{l}\text { Transient enlargement of CNS lesion and encephalopathy; necrosis, atrophy, calcification, } \\
\text { tumor inductions, CSF fistula, demyelination }\end{array}$ \\
\hline
\end{tabular}


Table 3. Surgical indication in patients with neurosarcoidosis [12,16-19].

\begin{tabular}{ll}
\hline Surgical Procedure & Indication \\
\hline Venticular drain insertion & $\begin{array}{l}\text { Symptomatic hydrocephalus } \\
\text { Meningeal/brain biopsy for tissue diagnosis epilepsy surgery neurosarcoid mass masquerad- } \\
\text { ing as neoplasm }\end{array}$ \\
Craniotomy & Treatment of isolated dilatation of third and fourth ventricles with patent aqueduct \\
Neuroendoscopy & Tissue biopsy, operative decompression of spinal sarcoidosis causing myeolpathy \\
Laminoplasty & Vertebral sarcoidosis resulting in spinal instability and neurological symptoms \\
Spine stabilization & Revascularization of large vessel stenosis caused by neurosarcoid vasculitis \\
Angioplasty & Lymph node tissue biopsy \\
\hline
\end{tabular}

\section{Anesthetic Implications}

Anesthetic management of patients with NS requires a thorough preoperative evaluation, search for functional impairment secondary to pulmonary, cardiac, hepatic or renal systemic involvement, and understanding interactions and complications of available as well as investigational treatment modalities.

Preoperative assessment should begin with a review of the patient's history and a focused neurological examination. Clinical presentation may vary based on the anatomic location of intracranial lesion. The anesthesiologist should be wary of the possibility of hypoventialtion due to respiratory center involvement or bilateral phrenic neuropathy [16]. Lower cranial nerve (IX, X) involvement although rare has been reported and poses an increased risk of aspiration [3]. All preexisting neurological deficits should be identified and the anesthetic plan formulated accordingly. Assessment may be limited due to diffuse encephalopathy, which may present as delirium, psychiatric disorders, memory disturbance and cognitive impairment. Hydrocephalus can develop which can be either communicating or obstructive and result in increased ICP [3]. Signs and symptoms of raised ICP should be identified. Preoperative sedation should be used cautiously, and drug induced hypoventilation avoided. Sarcoid granulomas have a predilection for the base of the brain and commonly involve the hypothalamus and pituitary gland resulting in hypothyroidism and hypoadrenalism [3]. Replacement hormone therapy should be continued in the perioperative period. The need for perioperative steroid supplementation should be determined on a case by case basis [17].

Counter-regulatory responses to hypoglycemia may be inadequate due to lack of compensatory elevations of catecholamine's, glucagon, cortisol, and growth hormone and merits consideration intraoperatively [18]. Hypotha- lamic dysfunction might impair temperature regulation [19]. Seizures can occur in upto $20 \%$ of patients with NS and thus potentially epileptogenic drugs (methohexitol, cis-atracurium) should be avoided [3]. Pharmacokinetic and pharmacodynamic interactions of antiepileptic drugs can cause shortening of the duration of action of nondepolarizing muscle relaxants [20]. Myopathic involvement is common but rarely symptomatic [21]. Nevertheless if neuromuscular blockers are used, neuromuscular monitoring is warranted. Water imbalance secondary to diabetes insipidus, and inappropriate antidiuretic hormone secretion (SIADH) can occur [3]. Urine output and serum electrolytes should be closely monitored in the perioperative period and appropriately replaced. Parenchymal brain disease, metabolic disturbances or seizures may result in delayed emergence.

\section{Conclusion}

In summary, NS is a serious and disabling disease which can virtually affect any part of the nervous system, posing a variety of clinical challenges. As NS is a rare disorder, our understanding of the management of this condition is by and large based on case reports or case series. While caring for these patients the anesthesiologist should not ignore the fact that other organ systems, in particular cardiac and pulmonary, may be involved and affect the conduct of anesthesia. A thorough assessment of the extent of involvement, resultant complications and their treatment is essential for the successful management of these patients.

\section{REFERENCES}

[1] B. A. Rybicki and M. C. Iannuzzi, "Epidemiology of Sarcoidosis: Recent Advances and Future Prospects," Seminars in Respiratory and Critical Care Medicine, Vol. 28, No. 1, 2007, pp. 22-35. doi:10.1055/s-2007-970331 
[2] R. K. Allen, R. E. Sellars and P. A. Sandstrom, "A Prospective Study of 32 Patients with Neurosarcoidosis," Sarcoidosis, Vasculitis and Diffuse Lung Diseases, Vol. 20, No. 2, 2003, pp. 118-125.

[3] V. Terushkin, B. J. Stern, M. A. Judson, M. Hagiwara, B. Pramanik, M. Sanchez, et al., "Neurosarcoidosis: Presentations and Management," Neurologist, Vol. 16, No. 1, 2010, pp. 2-15. doi:10.1097/NRL.0b013e3181c92a72

[4] A. V. Patel, D. E. Stickler and W. R. Tyor, "Neurosarcoidosis," Current Treatment Options in Neurology, Vol. 9, No. 3, 2007, pp. 161-168. doi:10.1007/BF02938405

[5] L. Ayala, D. B. Barber, M. R. Lomba and A. C. Able, "Intramedullary Sarcoidosis Presenting as Incomplete Paraplegia: Case Report and Literature Review,” Journal of Spinal Cord Medicine, Vol. 23, No. 2, 2000, pp. 96-99.

[6] D. A. Nowak and D. C. Widenka, "Neurosarcoidosis: A Review of Its Intracranial Manifestation,” Journal of Neurology, Vol. 248, No. 5, 2001, pp. 363-372. doi:10.1007/s004150170175

[7] E. E. Lower and K. L. Weiss, "Neurosarcoidosis," Clinics in Chest Medicine, Vol. 29, No. 3, 2008, pp. 475-492. doi:10.1016/j.ccm.2008.03.016

[8] I. B. Colvin, "Audiovestibular Manifestations of Sarcoidosis: A Review of the Literature," Laryngoscope, Vol. 116, No. 1, 2006, pp. 75-82. doi:10.1097/01.mlg.0000184580.52723.9f

[9] R. W. Byard, N. Manton and M. Tsokos, "Sarcoidosis and Mechanisms of Unexpected Death," Journal of Forensic Sciences, Vol. 53, No. 2, 2008, pp. 460-464. doi:10.1111/j.1556-4029.2008.00662.X

[10] S. Saleh, C. Saw, K. Marzouk and O. Sharma, "Sarcoidosis of the Spinal Cord: Literature Review and Report of Eight Cases," Journal of the National Medical Association, Vol. 98, No. 6, 2006, pp. 965-976.

[11] K. Oe, M. Doita, H. Miyamoto, F. Kanda, M. Kurosaka and M. Sumi, "Is Extensive Cervical Laminoplasty an Effective Treatment for Spinal Cord Sarcoidosis Combined with Cervical Spondylosis?” European Spine Journal, Vol. 18, No. 4, 2009, pp. 570-576. doi:10.1007/s00586-009-0891-2

[12] G. Galassi, M. Gibertoni, A. Mancini, R. Nemni, G. Volpi, E. Merelli and G. Vacca, "Sarcoidosis of the Peripheral Nerve: Clinical, Electrophysiological and Histological Study of Two Cases,” European Neurology, Vol. 23, No. 6, 1984, pp. 459-465. doi:10.1159/000115728
[13] T. F. Scott, K. Yandora, A. Valeri, C. Chieffe and C. Schramke, "Aggressive Therapy for Neurosarcoidosis: Long-Term Follow-Up of 48 Treated Patients,” Archives of Neurology, Vol. 64, No. 5, 2007, pp. 691-696. doi:10.1001/archneur.64.5.691

[14] J. P. Zajicek, N. J. Scolding, O. Foster, M. Rovaris, J. Evanson, I. F. Moseley, J. W. Scadding, E. J. Thompson, V. Chamoun, D. H. Miller, W. I. McDonald and D. Mitchell, "Central Nervous System Sarcoidosis: Diagnosis and Management,” Quarterly Journal of Medicine, Vol. 92, No. 2, 1999, pp. 103-117. doi:10.1093/qimed/92.2.103

[15] H. Akhondi, S. Barochia, B. Holmstrom and M. J. Williams, "Hydrocephalus as a Presenting Manifestation of Neurosarcoidosis,” Southern Medical Journal, Vol. 96, No. 4, 2003, pp. 403-406. doi:10.1097/01.SMJ.0000056648.43258.C0

[16] L. R. Robinson, R. Brownsberger and G. Raghu, "Respiratory Failure and Hypoventilation Secondary to Neurosarcoidosis," American Journal of Respiratory and Critical Care Medicine, Vol. 157, No. 4, 1998, pp. 13161318.

[17] S. L. Yong, P. Marik, M. Esposito and P. Coulthard, "Supplemental Perioperative Steroids for Surgical Patients with Adrenal Insufficiency," Cochrane Database of Systematic Reviews, 2009, Article ID: CD005367.

[18] F. Fery, L. Plat, P. van de Borne, E. Cogan and J. Mockel, "Impaired Counterregulation of Glucose in a Patient with Hypothalamic Sarcoidosis,” New England Journal of Medicine, Vol. 340, No. 11, 1999, pp. 852-856. doi:10.1056/NEJM199903183401105

[19] M. Jefferson, "Sarcoidosis of the Nervous System," Brain, Vol. 80, No. 4, 1957, pp. 540-556. doi:10.1093/brain/80.4.540

[20] A. Richard, F. Girard, D. C. Girard, D. Boudreault, P. Chouinard, R. Moumdjian, B. A. outhilier, M. Ruel, J. Couture and F. Varin, "Cisatracurium-Induced Neuromuscular Blockade Is Affected by Chronic Phenytoin or Carbamazepine Treatment in Neurosurgical Patients," Anesthesia \& Analgesia, Vol. 100, No. 2, 2005, pp. 538544. doi:10.1213/01.ANE.0000143333.84988.50

[21] M. M. Jamal, A. M. Cilursu and E. L. Hoffman, "Sarcoidosis Presenting as Acute Myositis. Report and Review of the Literature,” Journal of Rheumatology, Vol. 15, No. 12, 1988, pp. 1868-1871. 